\title{
Crosslinking and Mechanical Properties Significantly Influence Cell Attachment, Proliferation, and Migration Within Collagen Glycosaminoglycan Scaffolds
}

\author{
Matthew G. Haugh, B.A., B.A.I., Ph.D., ${ }^{1,2}$ Ciara M. Murphy, B.Sc., ${ }^{1}$ Ross C. McKiernan, B.Sc., \\ Cornelia Altenbuchner, M.S., ${ }^{1,3}$ and Fergal J. O'Brien, B.A., B.A.I., Ph.D.1,2
}

Crosslinking and the resultant changes in mechanical properties have been shown to influence cellular activity within collagen biomaterials. With this in mind, we sought to determine the effects of crosslinking on both the compressive modulus of collagen-glycosaminoglycan scaffolds and the activity of osteoblasts seeded within them. Dehydrothermal, 1-ethyl-3-3-dimethyl aminopropyl carbodiimide and glutaraldehyde crosslinking treatments were first investigated for their effect on the compressive modulus of the scaffolds. After this, the most promising treatments were used to study the effects of crosslinking on cellular attachment, proliferation, and infiltration. Our experiments have demonstrated that a wide range of scaffold compressive moduli can be attained by varying the parameters of the crosslinking treatments. 1-Ethyl-3-3-dimethyl aminopropyl carbodiimide and glutaraldehyde treatments produced the stiffest scaffolds (fourfold increase when compared to dehydrothermal crosslinking). When cells were seeded onto the scaffolds, the stiffest scaffolds also showed increased cell number and enhanced cellular distribution when compared to the other groups. Taken together, these results indicate that crosslinking can be used to produce collagen-glycosaminoglycan scaffolds with a range of compressive moduli, and that increased stiffness enhances cellular activity within the scaffolds.

\section{Introduction}

$\mathrm{C}^{\mathrm{c}}$ OLLAGEN-GLYCOSAMINOGLYCAN (CG) scaffolds were originally developed for skin regeneration and have since shown great potential in many areas of tissue engineering, including nerve, cartilage, and bone. ${ }^{1-7}$ Collagen is particularly attractive for tissue engineering due to its excellent biocompatibility, degradation into physiological end-products, and suitable interaction with cells and other macromolecules. ${ }^{8}$ Despite these advantages, the relatively weak mechanical properties of collagen may limit their use as a scaffold. Mechanical properties are an important factor when designing a scaffold for use in tissue engineering, as the scaffold must have suitable characteristics to facilitate in vitro handling and withstand the in vivo environment. In addition, there is a growing body of work demonstrating how the stiffness of a scaffold can direct cellular activity such as attachment and differentiation. ${ }^{9-12}$ Thus, scaffold stiffness can further determine the functionality of a tissue-engineered construct through mechano-transductive pathways. Crosslinking is a technique that is often used to enhance the mechanical properties of collagen-based biomaterials by forming bonds between collagen molecules. ${ }^{13-15}$ These bonds strengthen collagen fibers by preventing the long rod-like molecules from sliding past each other under stress. ${ }^{16}$ Due to the interaction between mechanical properties and biological activity, it is of interest to determine the effects of crosslinking treatments on both the mechanical properties of collagen scaffolds and the activity of cells within the crosslinked scaffolds.

Crosslinking treatments can be split into two main groups: chemical crosslinking and biophysical crosslinking. Examples of chemical methods include the use of glutaraldehyde (GTA) and various carbodiimides, whereas biophysical methods include the use of UV light and dehydrothermal (DHT) crosslinking. DHT treatment involves subjecting collagen to increased temperature $\left(>98^{\circ} \mathrm{C}\right)$ while under vacuum. This removes water from the collagen molecules, resulting in the formation of intermolecular crosslinks through condensation reactions (Fig. 1). An advantage of DHT crosslinking is that it does not involve the use of cytotoxic reagents. 1-Ethyl-3-3-dimethyl aminopropyl carbodiimide (EDAC) and GTA are two of the most commonly used chemical crosslinking agents. These two chemicals

\footnotetext{
${ }^{1}$ Department of Anatomy, Royal College of Surgeons in Ireland, Dublin, Ireland.

${ }^{2}$ Trinity Centre for Bioengineering, Department of Mechanical Engineering, Trinity College Dublin, Dublin, Ireland.

${ }^{3}$ Department of Mechanical Engineering, University of Applied Science Regensburg, Regensburg, Germany.
} 
A

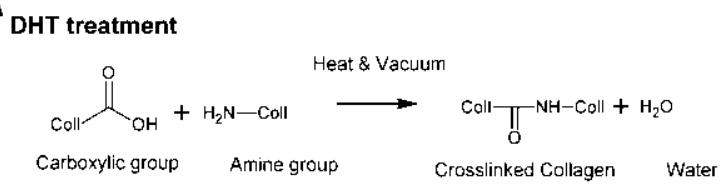

B EDAC structure \& crosslinking mechanism $_{\text {. }}$<smiles>CCN=C=NCCCN(C)C</smiles><smiles>O=C1CCC(=O)N1O</smiles>

Carboxylic group
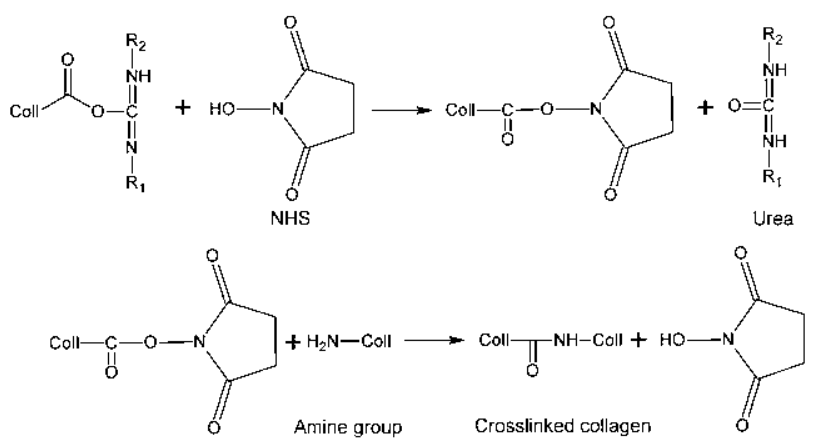

C GTA structure and crosslinking mechanism $_{\text {. }}$

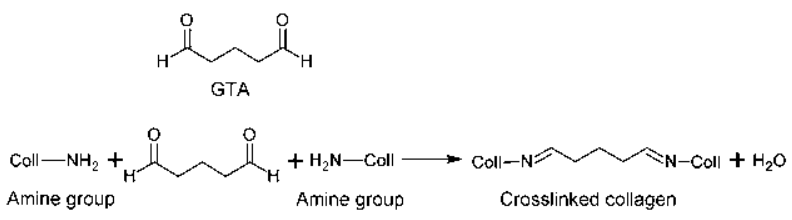

FIG. 1. The structure and crosslinking mechanisms of the treatments used in this study. (A) DHT treatment results in crosslink formation between adjacent carboxylic and amine groups through a condensation reaction. (B) EDAC and $\mathrm{N}$ hydroxysuccinimide (NHS) catalyze a reaction between adjacent carboxylic and amine groups to form crosslinks. EDAC first activates a carboxylic group, which then reacts with NHS to produce an NHS-activated carboxylic acid group and urea. Finally, the NHS-activated carboxylic acid group reacts with a free amine group to produce a crosslink. (C) GTA forms long polymer chains that react with amine groups to form crosslinks. DHT, dehydrothermal; EDAC, 1-ethyl-3-3-dimethyl aminopropyl carbodiimide; GTA, glutaraldehyde.

work in distinctly different fashions. EDAC forms "zero length" crosslinks, which are chemically identical to those formed during DHT treatment, whereas GTA crosslinks take the form of long polymer chains (Fig. 1). ${ }^{14,17}$ This means that EDAC is limited to crosslinking collagen molecules that are directly adjacent to each other $(1 \mathrm{~nm})$, whereas GTA can crosslink molecules that are separated by a distance. ${ }^{18}$ The nature of this chemical crosslinking involves the use of reagents that have the potential to cause cell death if they remain within the scaffold. It is therefore necessary to carry out experiments to determine if these chemicals have any cytotoxic side effects. EDAC crosslinking does not attach any harmful molecules to the scaffold, but it does need to be thoroughly washed out of the scaffolds before use as the chemical itself is toxic, as is urea, which is the by-product of the crosslinking reaction. ${ }^{17}$ GTA has the potential to leach out of the scaffold as it degrades, with potentially toxic repercussions for cells in the immediate area, ${ }^{19,20}$ although there is some debate as to whether it is cytotoxic on a clinically relevant level. ${ }^{15,21,22}$

Despite the widespread use of crosslinking treatments on collagen films, fibers, and scaffolds, a detailed analysis has not been carried out to determine how the process variables affect CG scaffold properties. Previous studies have concentrated on optimizing the effectiveness of individual crosslinking treatments, ${ }^{15,17,21,23}$ but a study directly comparing the effectiveness of several grades of different treatments has yet to be carried out. Additionally, many studies have investigated crosslinking of collagen fibers or films that may not be directly comparably to the crosslinking of a CG scaffold. ${ }^{13,23-25}$ Likewise, knowledge of the effect of crosslinking on the activity of cells seeded onto collagen scaffolds is incomplete.

The overall goal of this study was to determine the effects of crosslinking and the resulting changes in compressive modulus on cellular activity within CG scaffolds. Compressive testing was used to characterize the effect of crosslinking on mechanical properties as it allows comparison of the results to several previous studies ${ }^{15,26,27}$; additionally, compressive modulus has been shown to be the most relevant property when investigating cellular response to substrate properties. ${ }^{10,27}$ Based upon the results of the compressive testing, several groups were carried forward for cellular experiments. DHT treatment at $105^{\circ} \mathrm{C}$ for $24 \mathrm{~h}$ was chosen as a control, as it is the standard crosslinking treatment used in many studies. ${ }^{2,28,29}$ The scaffolds were seeded with an MC3T3 osteoblastic cell line, which has been used in several studies in our laboratory, thus allowing comparison to previous work. $^{28-31}$ The specific goals of this study were to investigate the effects of the crosslinking treatments on scaffold stiffness and then the seeding efficiency, proliferation, and spatial distribution of MC3T3-E1 pre-osteoblasts seeded onto CG scaffolds.

\section{Materials and Methods}

\section{Scaffold fabrication}

Scaffolds were produced by freeze-drying a CG suspension as described previously. ${ }^{32}$ Briefly, a suspension containing $0.5 \%(\mathrm{w} / \mathrm{v})$ collagen (Integra Life sciences) and $0.044 \% \quad(\mathrm{w} / \mathrm{v})$ chondroitin-6-sulfate (Sigma-Aldrich) was prepared in $0.05 \mathrm{M}$ acetic acid. This slurry was then freezedried using a freezing temperature of $-40^{\circ} \mathrm{C}$. This process produces a highly porous sheet of CG scaffold, with an average pore size of $120 \mu \mathrm{m} .^{32}$

\section{Crosslinking}

After freeze-drying cylindrical samples (Mechanical testing: $8 \mathrm{~mm}$ diameter $\times 4 \mathrm{~mm}$ height; Cell culture: $12.7 \mathrm{~mm}$ diameter $\times 4 \mathrm{~mm}$ height) were cut from the scaffold sheet. DHT treatment was carried out by placing the samples in an 
aluminium foil packet inside a vacuum oven (Vacucell 22; MMM) under a vacuum of 0.05 bar. To determine the effect of DHT parameters on scaffold performance, four different crosslinking temperatures were used: $105^{\circ} \mathrm{C}, 120^{\circ} \mathrm{C}, 150^{\circ} \mathrm{C}$, and $180^{\circ} \mathrm{C}$. To determine the effect of EDAC crosslinking, five different concentrations of EDAC (Sigma-Aldrich, Germany) were used: $0.6,3,6,48$, and $96 \mathrm{mM}$ EDAC per gram CG scaffold. These concentrations were prepared in $2 \mathrm{~mL}$ of sterile distilled water and the samples were crosslinked in 24-well plates for $24 \mathrm{~h}$. $\mathrm{N}$-Hydroxysuccinimide (NHS) is a catalyst that is commonly used with EDAC; a molar ratio of $2.5 \mathrm{M}$ EDAC/M N-hydroxysuccinimide was used for all EDAC crosslinking. ${ }^{24}$ GTA (Sigma-Aldrich) crosslinking was also carried out using five concentrations: $0.25,0.5,1.25,2.5$, and $3.75 \mathrm{mM}$ GTA per gram of CG scaffold. GTA crosslinking solutions were prepared in phosphate-buffered saline (PBS) and the samples were crosslinked in 24-well plates for $24 \mathrm{~h}$. After crosslinking the scaffolds were washed twice using sterile PBS (Sigma-Aldrich). The concentrations of EDAC and GTA are expressed relative to a gram of collagen throughout this article.

\section{Mechanical testing}

Compressive testing was used to determine the effect of crosslinking on the modulus of the scaffolds. Mechanical testing of scaffold samples was carried out using a mechanical testing machine (Z050; Zwick/Roell) fitted with a $5-\mathrm{N}$ load cell. Samples were prehydrated in PBS for $1 \mathrm{~h}$ before testing and all testing was carried out in a bath of PBS. For unconfined compression testing with impermeable, un-lubricated platens, samples of $8 \mathrm{~mm}$ diameter $(n=10$ per group) were cut from the scaffolds using a punch. Testing was conducted at a strain rate of $10 \% / \mathrm{min}$. The modulus was defined as the slope of a linear fit to the stress-strain curve over $2 \%-5 \%$ strain. ${ }^{26}$

\section{Cell culture}

MC3T3-E1 preosteoblasts (ATCC) were cultured in T175 culture flasks (Sarstedt) using $\alpha$-minimum essential media (Sigma-Aldrich) supplemented with 10\% fetal bovine serum (BioSera), 1\% L-glutamine (Sigma-Aldrich), and 2\% penicillin/ streptomycin (Sigma-Aldrich). Before seeding, cells (Passage 26) were detached using trypsin-ethylenediaminetetraacetic acid (Sigma-Aldrich) and suspended in supplemented medium at $10^{7}$ cells $/ \mathrm{mL}$. Crosslinked scaffolds were placed into 6-well tissue culture plates (Sarstedt). The top surface of each of the scaffolds was then seeded with $100 \mu \mathrm{L}$ of the cell suspension $\left(1 \times 10^{6}\right.$ cells $)$. The six-well plates were then placed in an incubator for $15 \mathrm{~min}$ to allow initial cellular attachment. The scaffolds were then turned over and the opposite surface was seeded with $100 \mu \mathrm{L}$ of the cell suspension and incubated for a further $15 \mathrm{~min}$. After the second incubation period, $5 \mathrm{~mL}$ of supplemented medium was added to each well and the plates were returned to the incubator. Both culture flasks and seeded scaffolds were cultured under standard conditions $\left(37^{\circ} \mathrm{C}, 5 \% \mathrm{CO}_{2}\right)$.

\section{Hoechst DNA assay}

Cell number was evaluated using the Hoechst 33258 DNA assay, which fluorescently labels double-stranded DNA
(Sigma-Aldrich), according to a previously published protocol. ${ }^{33}$ After 1, 2, and 7 days of incubation, seeded scaffolds ( $n=3$ per group) were washed twice using PBS to remove un-adheared cells before being flash-frozen in liquid nitrogen and stored at $-80^{\circ} \mathrm{C}$. Samples were then thawed and digested in papain (Sigma-Aldrich) for $1.5 \mathrm{~h}$ at $60^{\circ} \mathrm{C}$. Hoechst dye solution was then added to the digested samples and $210 \mu \mathrm{L}$ was then plated, in triplicate, into a 96-well plate. Fluorescence was then measured (excitation: $355 \mathrm{~nm}$; emission: $460 \mathrm{~nm}$ ) using a fluorescence spectrophotometer (Wallac Victor2; PerkinElmer Life Sciences). Readings were converted to cell number using a standard curve, with unseeded scaffolds used as blanks.

\section{Histology}

Histological analysis was used to evaluate the spatial distribution of cells within the scaffolds. After 1, 2, and 7 days of incubation, scaffold samples were placed into a solution of $10 \%$ formalin for $30 \mathrm{~min}$ and then embedded in paraffin wax using an automatic tissue processor (ASP300; Leica). All samples were sectioned longitudinally at a thickness of $10 \mu \mathrm{m}$ throughout the depth of the sample using a rotary microtome (Leica microtome; Leica). Sections were mounted on slides and stained using hematoxylin and eosin to evaluate spatial distribution of cells within each of the scaffolds. Digital images were recorded using a microscope (Optimphot2; Nikon).

\section{Statistical analysis}

Results are expressed in the figures as mean \pm standard deviation. One-way analysis of variance followed by pairwise multiple comparison procedures (Tukey test) was used to evaluate the effects of crosslinking on both the compressive modulus of CG scaffolds and cellular attachment. Statistical significance was declared at $p \leq 0.05$.

\section{Results}

\section{Effect of crosslinking on compressive modulus}

Increasing the temperature of DHT treatment was found to result in an increase in the mechanical properties. Compressive modulus increased twofold for scaffolds crosslinked at $180^{\circ} \mathrm{C}$ compared with that at $105^{\circ} \mathrm{C}(p<0.001$; Fig. 2A). However, as significant denaturation has been previously observed at $180^{\circ} \mathrm{C}$ using Fourier transform infrared microscopy $^{26}$ and crosslinking at $150^{\circ} \mathrm{C}$ produced a 1.9 -fold increase in modulus, the scaffolds carried forward to the cellular attachment experiments were crosslinked at $150^{\circ} \mathrm{C}$. Analysis of the mechanical testing after EDAC treatment revealed that compressive modulus increased with increasing EDAC concentration $(p<0.05$; Fig. 2B). A concentration of $96 \mathrm{mM}$ EDAC produced the stiffest scaffolds, with a compressive modulus of $1.8 \mathrm{kPa}$, representing a fourfold increase in modulus over the untreated scaffolds $(p<0.05)$. However, a concentration of $6 \mathrm{mM}$ EDAC produced a similarly significant increase in modulus when using one 16th the amount of EDAC. Therefore, both concentrations were used in the cellular attachment study, $96 \mathrm{mM}$ as it produced the stiffest scaffolds and $6 \mathrm{mM}$ as it was the most efficient treatment. Analysis of the mechanical testing after GTA treatment revealed that compressive modulus increased with 
A

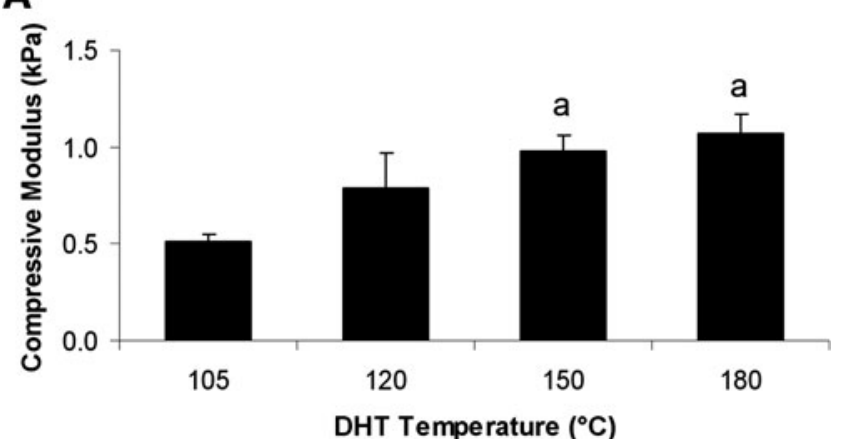

B
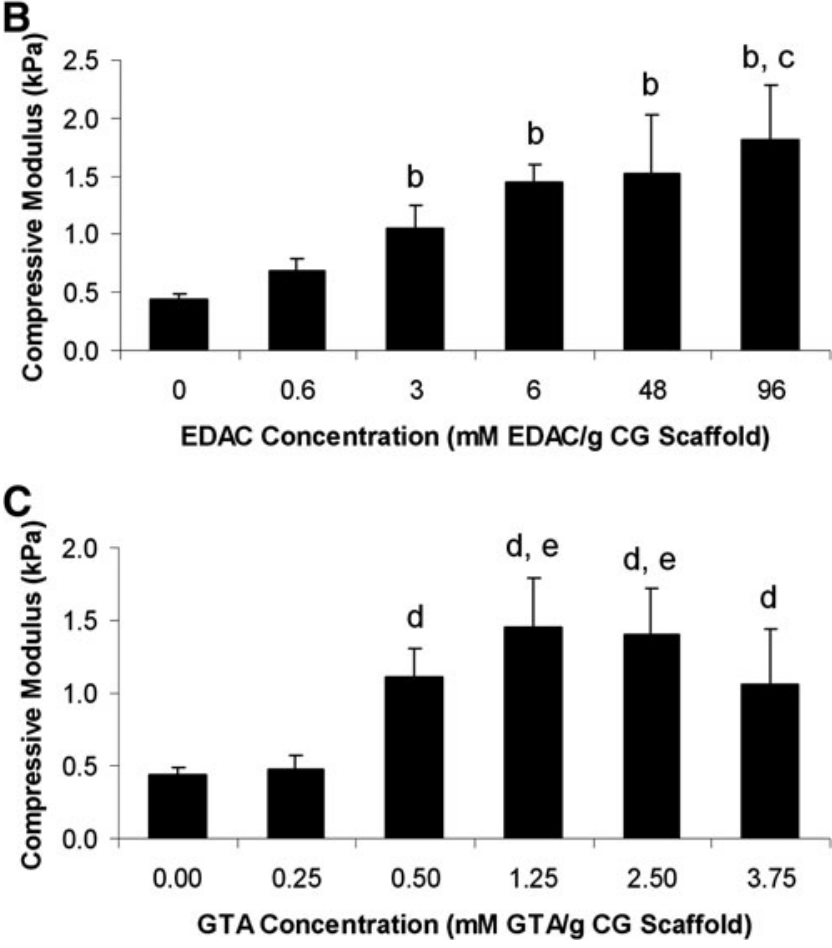

FIG. 2. The effect of various crosslinking treatments on the compressive modulus of the scaffolds (A) DHT, (B) EDAC, and (C) GTA. $N=10$ samples per group, ${ }^{a} p<0.05$ relative to $105^{\circ} \mathrm{C},{ }^{b} p<0.05$ relative to $0 \mathrm{mM}$ EDAC, ${ }^{c} p<0.05$ relative to all other groups, ${ }^{\mathrm{d}} p<0.05$ relative to $0 \mathrm{~g} \mathrm{GTA}$, and ${ }^{\mathrm{e}} p<0.05$ relative to $0.05 \mathrm{~g}$ GTA. CG, collagen-glycosaminoglycan.

concentration up to $1.25 \mathrm{mM}$ GTA ( $p<0.05$; Fig. $2 \mathrm{C}$ ). Further increases gave insignificant changes in modulus, with a reduction in modulus comparing $3.75 \mathrm{mM}$ GTA to $1.25 \mathrm{mM}$ GTA $(p>0.05)$. GTA crosslinking using $1.25 \mathrm{mM}$ produced the stiffest scaffolds, with a compressive modulus of $1.45 \mathrm{kPa}$. This was a threefold increase over the untreated scaffolds $(p<0.05)$, and consequently this concentration was used in the cellular attachment study.

\section{Effect of crosslinking on cell number within CG scaffolds}

The number of cells within the scaffolds was found to vary with the method of crosslinking used (Fig. 3A). The $6 \mathrm{mM}$ EDAC and $1.25 \mathrm{mM}$ GTA groups showed a significant in-

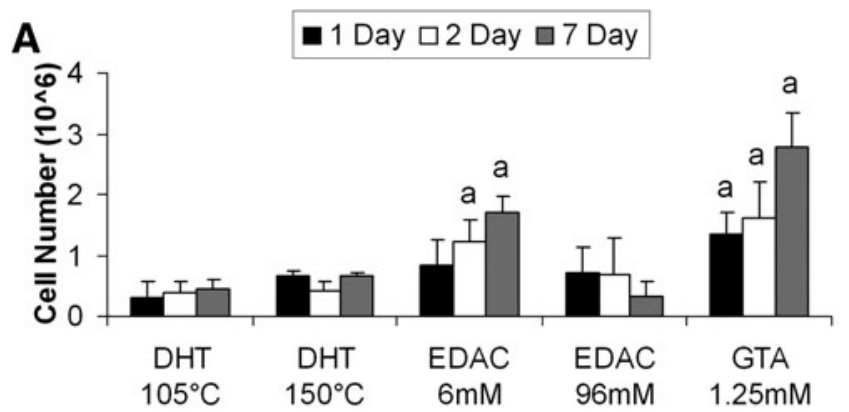

Crosslinking Treatment
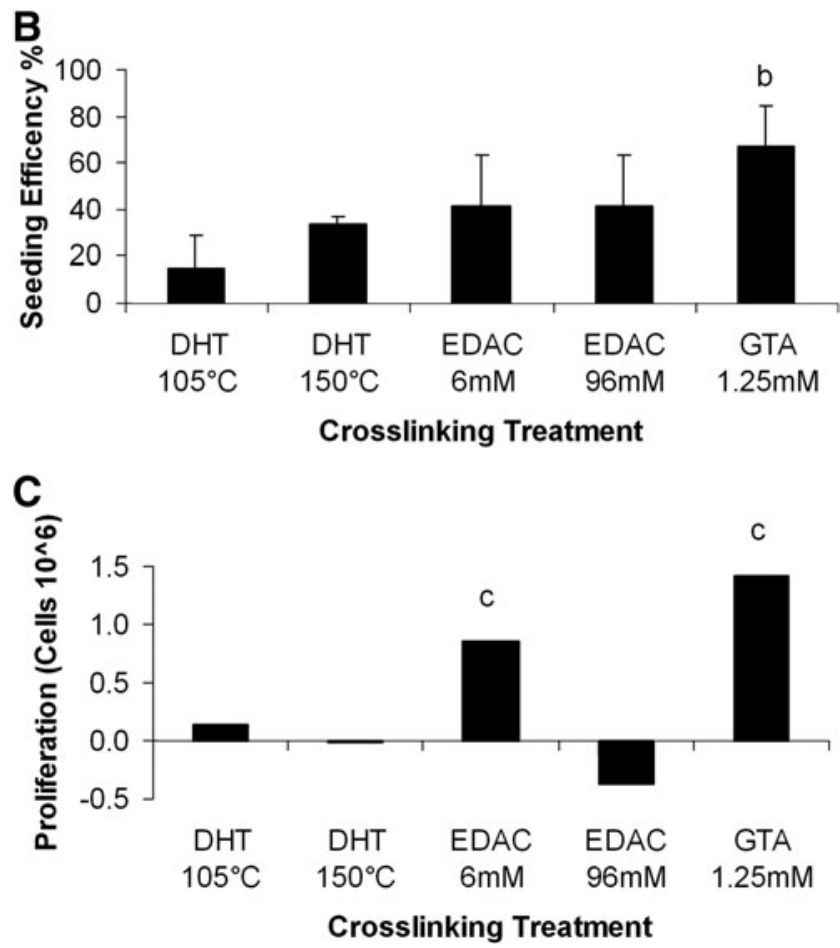

FIG. 3. The effect of crosslinking on (A) cell number within the scaffold at days 1, 2 and 7, (B) cell number at day 1 expressed as a percentage of seeded cell number, and (C) proliferation within the scaffolds as measured by subtracting the cell number at day 1 from the cell number at day 7. $N=3$ per group, ${ }^{\mathrm{a}} p<0.05$ relative to $105^{\circ} \mathrm{C},{ }^{\mathrm{b}} p<0.05$ relative to all other groups, and ${ }^{c} p<0.05$ significant change in cell number between days 1 and 7 .

crease in cell number over the standard, DHT $105^{\circ} \mathrm{C}$-treated scaffolds at all time points $(p<0.05)$. In particular, GTAcrosslinked scaffolds had a significantly higher cell number than all other treatment groups, showing a fivefold increase in cell number compared to the standard DHT $105^{\circ} \mathrm{C}$ crosslinked scaffolds $(p<0.001)$.

\section{Initial cell adhesion to CG scaffolds and proliferation}

To better interpret the data, the effect of crosslinking on seeding efficiency was measured by expressing the number of cells attached after 1 day as a percentage of the number of cells initially seeded $\left(2 \times 10^{6}\right.$ cells, Fig. 3B). The GTA-treated group had the highest seeding efficiency, with $70 \%$ of seeded cells attached after 1 day. This represents a 4.5-fold increase 
over the DHT $105^{\circ} \mathrm{C}$ treatment $(p<0.001)$. Cellular proliferation upon the scaffolds was measured using the difference between the cell number at 1 and 7 days (Fig. 3C). Significant levels of proliferation were found in both the $6 \mathrm{mM}$ EDAC and GTA crosslinking groups $(p<0.05)$. In particular, a twofold increase in cell number was found in the GTAcrosslinked group $(p<0.001)$. A nonstatistically significant reduction in cell number was found in the $96 \mathrm{mM}$ EDACcrosslinked group.

\section{Cell infiltration}

Histology was used to determine the effect of crosslinking on the spatial distribution of cells within the CG scaffolds at 1,2 , and 7 days. Sections at the day 7 time point are shown in Figure 4 to illustrate the differences in cellular infiltration between the groups. As the sections from the DHT $150^{\circ} \mathrm{C}$ and $96 \mathrm{mM}$ EDAC groups were similar to the DHT $105^{\circ} \mathrm{C}$ group, they have been omitted. All treatment groups show cellular attachment at the edge of the scaffolds at 1 and 7 days. There was no evidence of cellular infiltration into the center of the scaffolds after 7 days in the DHT (Fig. 4B) and $96 \mathrm{mM}$ EDAC groups (not shown). However, both the $6 \mathrm{mM}$ EDAC and GTA crosslinking groups show notable levels of cellular infiltration at the center of the scaffolds after 7 days of culture (Fig. 4D, F); this was more pronounced in the GTA group.

\section{Discussion}

The aim of this study was to develop a series of CG scaffolds with a range of compressive moduli and to determine the effects of compressive modulus on cellular behavior. We used osteoblasts for cellular characterization as we have a specific interest in scaffolds for bone repair; however, the results obtained have led to the production of a series of characterized scaffold variants that can be adapted for other tissue engineering applications or used to expand our understanding of the interactions between scaffold properties and cellular activity. Our experiments have demonstrated that a wide range of compressive moduli can be attained by varying the parameters of the crosslinking treatments (Fig. 2). The stiffest scaffolds were produced using $96 \mathrm{mM}$ EDAC and $1.25 \mathrm{mM}$ GTA, which had compressive moduli of $1.8 \mathrm{kPa}$ and $1.4 \mathrm{kPa}$, respectively. When cells were seeded onto the scaffolds, the $6 \mathrm{mM}$ EDAC and $1.25 \mathrm{mM}$ GTAcrosslinked scaffolds showed increased cell number and enhanced cellular distribution when compared to the other crosslinking treatments (Figs. 3 and 4). Taken together, these results indicate that crosslinking can be used effectively to produce CG scaffolds with a range of compressive moduli, and that increased stiffness enhances cellular activity within the scaffolds.

The methods used in this study specify the effects of several crosslinking treatments at a range of different
$\mathrm{DHT} 105^{\circ} \mathrm{C}$
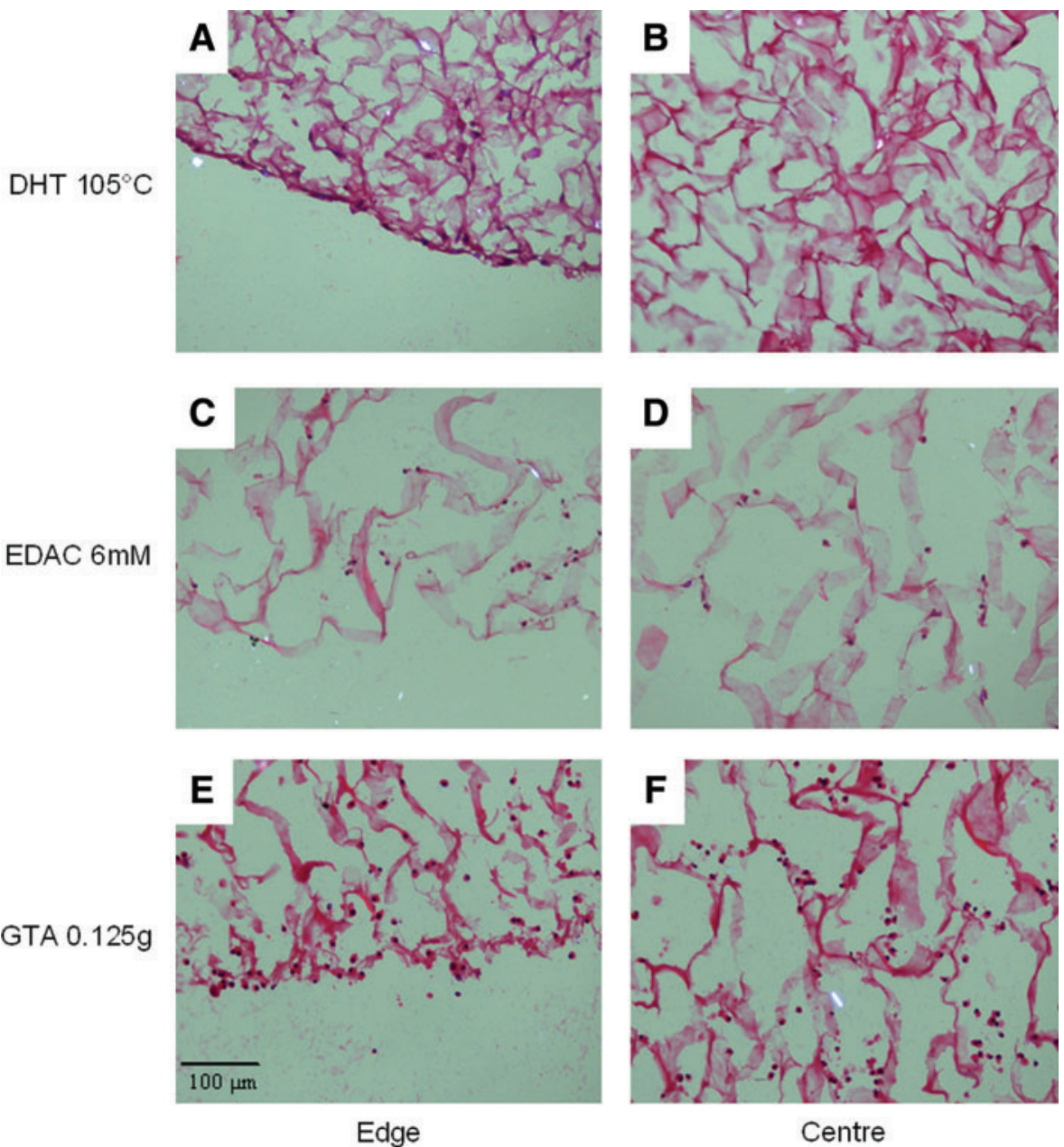

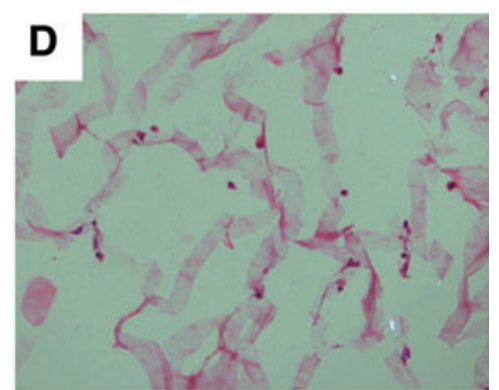

Centre

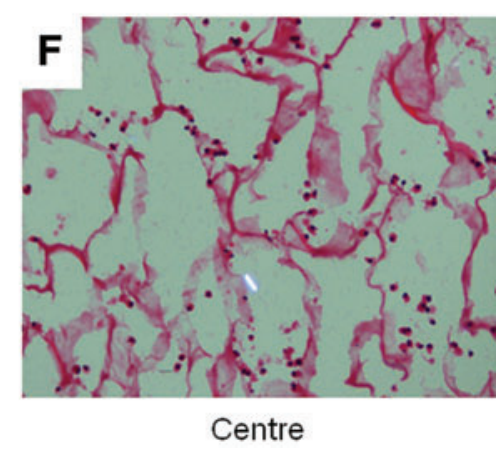

Edge

GTA $0.125 \mathrm{~g}$ GTA $0.125 g$
FIG. 4. Effect of crosslinking on cell infiltration and distribution within CG scaffolds after 7 days. Scaffolds were stained with hematoxylin and eosin: (A) edge of DHT $105^{\circ} \mathrm{C}$, (B) center of DHT $105^{\circ} \mathrm{C},(\mathbf{C})$ edge of EDAC $6 \mathrm{mM}$, (D) center of EDAC $6 \mathrm{mM}$, (E) edge of GTA $0.125 \mathrm{~g}$, and (F) center of the GTA $0.125 \mathrm{~g}$. Collagen scaffold is stained pink and cell nuclei a deep purple. Infiltration of cells into the center of the scaffolds and increased cell number can be seen in the stiffer EDAC- and GTA-crosslinked groups. Color images available online at www.liebertonline .com/ten. 
concentrations that can be directly related to the collagen content of the scaffolds. The majority of the work previously published has neglected to give the molar ratios of the crosslinking agents to the weight of collagen treated, ${ }^{15,17,34,35}$ preventing the results from being used in an effective manner as the exact crosslinking conditions cannot be reproduced. This information is particularly useful when the relationship between cytotoxicity and the concentration of a crosslinking agent is of interest. In this study we use compressive testing to determine the effects of crosslinking upon mechanical properties, whereas several studies use tensile testing. ${ }^{13,35,36}$ However, previous work has shown that the compressive properties are of greatest interest when studying the effects of substrate properties on cellular activity such as cell-mediated contraction, attachment, and differentiation. ${ }^{10,27}$ Taking into account these studies and the material demands of tensile testing, it was decided that compressive testing would provide sufficient data for mechanical characterization of the crosslinked scaffolds.

Harley et al. also examined the effectiveness of EDAC crosslinking of CG scaffolds and reported a compressive modulus of $1.497 \mathrm{kPa}$ when treating scaffolds with $6 \mathrm{mM}$ EDAC. $^{27}$ This compares well with our result of $1.442 \mathrm{kPa}$ with scaffolds crosslinked at the same molar ratio. While Olde Damink et al. reported that the optimal increase in shrinkage temperature and crosslinks formed was achieved using $6 \mathrm{mM}$ of EDAC per gram of collagen, our results show the highest compressive modulus at $96 \mathrm{mM} .{ }^{24}$ EDAC forms crosslinks between carboxylic and amine groups (Fig. 1), which are present in the scaffolds at concentrations of $1.264 \mathrm{mM}$ and $0.312 \mathrm{mM}$ per gram of CG scaffold, respectively. ${ }^{24,37}$ The maximum number of crosslinks is therefore limited by the amine group content. However, due to the close positional alignment needed for EDAC crosslinking it may be required to activate all the carboxylic groups present to complete crosslinking. Therefore, a concentration of $1.264 \mathrm{mM}$ EDAC would be required to form the theoretical maximum number of crosslinks. Our results show that $96 \mathrm{mM}$ EDAC produced the maximum compressive modulus; the difference between this amount and the maximum theoretical concentration required may be explained by the fact that EDAC has been observed to react with the urea formed as a by product of the crosslinking reaction. ${ }^{38}$ This reaction with urea may also explain the reduction in the efficiency of the crosslinking reaction above $6 \mathrm{mM}$. GTA forms crosslinks between two amide groups (Fig. 1), and this would limit the theoretical maximum number of crosslinks to $0.156 \mathrm{mM}$ per gram of CG scaffold. ${ }^{14}$ However, as GTA has the ability to form polymer chains, estimation of the theoretical concentration of GTA needed to form the maximum number of crosslinks and the modulus of GTA treated CG scaffolds, while of interest, would be beyond the scope of this study.

The most favorable modulus was attained using $96 \mathrm{mM}$ EDAC and $1.25 \mathrm{mM}$ GTA. Crosslinking using these treatments resulted in a three- to fourfold increase in compressive modulus (Fig. 2B, C). In addition, $6 \mathrm{mM}$ EDAC crosslinking imparted a modulus comparable to the $96 \mathrm{mM}$ EDAC group while making efficient use of the crosslinking reagent $(4 / 5$ ths of the highest modulus was attained using 1/16th the amount of EDAC), this proved to be of importance when the cytotoxicity of the treatment was evaluated. The $6 \mathrm{mM}$
EDAC and 1.25 mM GTA treatment groups show enhanced seeding efficiency, proliferation, and infiltration (Figs. 3 and 4). In particular, GTA-crosslinked scaffolds showed a 6.4-fold increase in cell number at 7 days when compared to the standard DHT $105^{\circ} \mathrm{C}$ group. Additionally, the proliferation data correlate with infiltration as the $6 \mathrm{mM}$ EDAC and $1.25 \mathrm{mM}$ GTA groups are the only groups to show infiltration into the center of the scaffolds after 7 days. The difference in the proliferation between the $6 \mathrm{mM}$ EDAC group and the $1.25 \mathrm{mM}$ GTA group is likely to be a result of higher cell number at day 1 due to superior seeding efficiency. Examining the data from the cellular attachment within the DHT-treated scaffolds, we report a $15 \%$ seeding efficiency at $24 \mathrm{~h}$ upon the standard scaffolds compared to $40 \%$ reported by $\mathrm{O}^{\prime}$ Brien et al. ${ }^{29}$ However, the scaffolds in the $\mathrm{O}^{\prime}$ Brien study were seeded dry, which would aid infiltration as the cell suspension would not have to displace any fluid already within the scaffolds. The DHT-treated scaffolds in this study were seeded in a hydrated state to enable a direct comparison to chemical treatments that required the scaffolds to be hydrated at the seeding stage. This limitation could have been overcome through freeze-drying the chemically crosslinked scaffolds to dry them, though it is likely that this would have caused some damage to the structure of the scaffolds.

Previous work has postulated that cell-mediated contraction may reduce proliferation of chondrocytes. ${ }^{15}$ In this study, the only groups observed to show any cell-mediated contraction were the groups treated using DHT treatment. Therefore, the lack of proliferation in these groups may be caused by contraction of the scaffold structure, which among other things causes a collapse of the scaffold pore structure reducing the diffusion of nutrients and waste products within the scaffold. The increased modulus of GTA and EDAC-crosslinked scaffolds enabled them to retain their porous structure, allowing proliferation and infiltration within these groups. Further, cells have been shown to have the capability to react to the mechanical properties of the material to which they are attached. ${ }^{9,10}$ This has been postulated to be related to the tension or pull generated through actomyosin filaments acting through the integrin-actin coupling at focal adhesion complexes. ${ }^{39,40}$ Further adding to this hypothesis, Engler et al. have shown that nonmuscle myosin plays a role in the sensitivity of cells to substrate stiffness. ${ }^{10}$ Thus, the mechanical properties of the scaffolds may influence cellular attachment and proliferation through mechanotransductive pathways in addition to their effect on physical parameters caused by the retention of pore architecture. However, one caveat to note is that although the $1.25 \mathrm{mM}$ GTA and $6 \mathrm{mM}$ EDAC-crosslinked scaffolds have comparable moduli, the GTA-crosslinked scaffolds show superior seeding efficiency. This suggests that the presence of GTA crosslinks, in some way, positively influences seeding and cellular attachment. Crosslinking consumes hydrophilic amino groups and therefore causes a reduction in collagen hydrophilicity. Previous studies have shown that EDAC causes a larger reduction in the hydrophilic nature of collagen films compared to GTA. ${ }^{25}$ Any increase in scaffold hydrophobicity might reduce the volume of cell suspension retained within the scaffold during seeding, thus potentially explaining the poorer cellular retention in the EDAC-treated in comparison to the GTA-treated groups. 
The $96 \mathrm{mM}$ EDAC group did not perform as well as the other chemical crosslinking groups, showing a reduction in cell number after 7 days. This may be evidence of the cytotoxic effects of EDAC at high concentrations. Although EDAC is not incorporated into the scaffold when it forms crosslinks, the by-product of this reaction is urea, which is cytotoxic if left within the scaffolds. Previous work has found a reduction in cellular activity when high concentrations of EDAC are used to crosslink collagen scaffolds. ${ }^{17}$ The authors postulated that it becomes more difficult to wash out the resultant urea and unused EDAC when high concentrations of EDAC are used. We found no evidence of cytotoxicity caused by GTA crosslinking. GTA is integrated into the crosslinks that are formed, and un-reacted GTA may also remain within the scaffold after crosslinking. Previous studies have found some evidence of cytotoxicity, causing reduced proliferation, with cells cultured on GTA-crosslinked CG scaffolds. ${ }^{15}$ Contrary to this, we found an increase in cellular activity with GTA-crosslinked scaffolds. However, there is still the potential for damage to the cells to occur as GTA is released while the scaffold degrades, although some in vivo studies show no evidence of adverse effects. ${ }^{21}$ In the context of bone tissue engineering, there are differing reports in the literature on the relationship between GTA crosslinking and mineralization. GTA crosslinking has been shown in some studies to induce calcification in vivo. ${ }^{34,41}$ However, there are reports that GTA may also compromise mineralization in CG scaffolds. ${ }^{22}$ Long-term cell culture experiments in osteogenic media are needed to clarify these findings.

\section{Conclusion}

When considering a crosslinking method for scaffolds used in a particular tissue engineering application, the effects of the various treatments on cellular activity are just as important as the effects on mechanical properties. The results of this study show that EDAC and GTA crosslinking can be used to substantially improve the compressive modulus of CG scaffolds and that cellular activity within the scaffolds was enhanced as a result of the increased stiffness produced through crosslinking. GTA-crosslinked scaffolds show the greatest potential for tissue engineering, despite not possessing the highest compressive modulus, having the ability to support 6.4 times as many cells as the standard DHTcrosslinked scaffolds $(p<0.05)$. The enhanced cellular activity upon GTA- and EDAC-treated scaffolds, coupled with improved compressive stiffness, increases their suitability for use both in vitro and in vivo.

\section{Acknowledgments}

The authors acknowledge the Science Foundation Ireland, President of Ireland Young Researcher Award (04/Y11/ B531), for funding. R.C. McKiernan was funded by the HRB in Ireland under Grant No PHD/2007/11. Collagen materials were provided by Integra Life Sciences, Inc., through a material transfer agreement.

\section{Disclosure Statement}

No competing financial interests exist.

\section{References}

1. Yannas, I.V., and Burke, J.F. Design of an artificial skin. I. Basic design principles. J Biomed Mater Res 14, 65, 1980.

2. Yannas, I.V., Burke, J.F., Gordon, P.L., Huang, C., and Rubenstein, R.H. Design of an artificial skin. II. Control of chemical composition. J Biomed Mater Res 14, 107, 1980.

3. Dagalakis, N., Flink, J., Stasikelis, P., Burke, J.F., and Yannas, I.V. Design of an artificial skin. Part III. Control of pore structure. J Biomed Mater Res 14, 511, 1980.

4. Burke, J.F., Yannas, I.V., Quinby, W.C., Jr., Bondoc, C.C., and Jung, W.K. Successful use of a physiologically acceptable artificial skin in the treatment of extensive burn injury. Ann Surg 194, 413, 1981.

5. Chamberlain, L.J., Yannas, I.V., Hsu, H.P., Strichartz, G., and Spector, M. Collagen-GAG substrate enhances the quality of nerve regeneration through collagen tubes up to level of autograft. Exp Neurol 154, 315, 1998.

6. Vickers, S.M., Squitieri, L.S., and Spector, M. Effects of crosslinking type II collagen-GAG scaffolds on chondrogenesis in vitro: dynamic pore reduction promotes cartilage formation. Tissue Eng 12, 1345, 2006.

7. Tierney, C.M., Haugh, M.G., Liedl, J., Mulcahy, F., Hayes, B., and $\mathrm{O}^{\prime}$ Brien, F.J. The effects of collagen concentration and crosslink density on the biological, structural and mechanical properties of collagen-GAG scaffolds for bone tissue engineering. J Mech Behav Biomed Mater 2, 202, 2009.

8. Geiger, M., Li, R.H., and Friess, W. Collagen sponges for bone regeneration with rhBMP-2. Adv Drug Deliv Rev 55, 1613, 2003.

9. Engler, A., Bacakova, L., Newman, C., Hategan, A., Griffin, M., and Discher, D. Substrate compliance versus ligand density in cell on gel responses. Biophys J 86(1 Pt 1), 617, 2004.

10. Engler, A.J., Sen, S., Sweeney, H.L., and Discher, D.E. Matrix elasticity directs stem cell lineage specification. Cell 126, 677, 2006.

11. Yeung, T., Georges, P.C., Flanagan, L.A., Marg, B., Ortiz, M., Funaki, M., et al. Effects of substrate stiffness on cell morphology, cytoskeletal structure, and adhesion. Cell Motil Cytoskeleton 60, 24, 2005.

12. Engler, A.J., Richert, L., Wong, J.Y., Picart, C., and Discher, D.E. Surface probe measurements of the elasticity of sectioned tissue, thin gels and polyelctrolyte multilayer films: correlations between substrate stiffness and cell adhesion. Surf Sci 570, 142, 2004.

13. Weadock, K., Olson, R.M., and Silver, F.H. Evaluation of collagen crosslinking techniques. Biomater Med Devices Artif Organs 11, 293, 1983.

14. Khor, E. Methods for the treatment of collagenous tissues for bioprostheses. Biomaterials 18, 95, 1997.

15. Lee, C.R., Grodzinsky, A.J., and Spector, M. The effects of cross-linking of collagen-glycosaminoglycan scaffolds on compressive stiffness, chondrocyte-mediated contraction, proliferation and biosynthesis. Biomaterials 22, 3145, 2001.

16. Bailey, A.J., Light, N.D., and Atkins, E.D. Chemical crosslinking restrictions on models for the molecular organization of the collagen fibre. Nature 288, 408, 1980.

17. Powell, H.M., and Boyce, S.T. EDC cross-linking improves skin substitute strength and stability. Biomaterials 27, 5821, 2006.

18. Zeeman, R., Dijkstra, P.J., van Wachem, P.B., van Luyn, M.J., Hendriks, M., Cahalan, P.T., et al. Successive epoxy and carbodiimide cross-linking of dermal sheep collagen. Biomaterials 20, 921, 1999. 
19. Wiebe, D., Megerman, J., L'Italien, G.J., and Abbott, W.M. Glutaraldehyde release from vascular prostheses of biologic origin. Surgery 104, 26, 1988.

20. Gendler, E., Gendler, S., and Nimni, M.E. Toxic reactions evoked by glutaraldehyde-fixed pericardium and cardiac valve tissue bioprosthesis. J Biomed Mater Res 18, 727, 1984

21. Kikuchi, M., Matsumoto, H.N., Yamada, T., Koyama, Y., Takakuda, K., and Tanaka, J. Glutaraldehyde cross-linked hydroxyapatite/collagen self-organized nanocomposites. Biomaterials 25, 63, 2004.

22. Chen, D.C., Lai, Y.L., Lee, S.Y., Hung, S.L., and Chen, H.L. Osteoblastic response to collagen scaffolds varied in freezing temperature and glutaraldehyde crosslinking. J Biomed Mater Res A 80, 399, 2007.

23. Ruijgrok, J.M., de Wijn, J.R., and Boon, M.E. Glutaraldehyde crosslinking of collagen: effects of time, temperature, concentration and presoaking as measured by shrinkage temperature. Clin Mater 17, 23, 1994.

24. Olde Damink, L.H., Dijkstra, P.J., van Luyn, M.J., van Wachem, P.B., Nieuwenhuis, P., and Feijen, J. Cross-linking of dermal sheep collagen using a water-soluble carbodiimide. Biomaterials 17, 765, 1996.

25. Ber, S., Torun Kose, G., and Hasirci, V. Bone tissue engineering on patterned collagen films: an in vitro study. Biomaterials 26, 1977, 2005.

26. Haugh, M.G., Jaasma, M.J., and O'Brien, F.J. The effect of dehydrothermal treatment on the mechanical and structural properties of collagen-GAG scaffolds. J Biomed Mater Res A 89, 363, 2009.

27. Harley, B.A., Leung, J.H., Silva, E.C., and Gibson, L.J. Mechanical characterization of collagen-glycosaminoglycan scaffolds. Acta Biomater 3, 463, 2007.

28. Tierney, C.M., Jaasma, M.J., and O'Brien, F.J. Osteoblast activity on collagen-GAG scaffolds is affected by collagen and GAG concentrations. J Biomed Mater Res A 91, 92, 2009.

29. O'Brien, F.J., Harley, B.A., Yannas, I.V., and Gibson, L.J. The effect of pore size on cell adhesion in collagen-GAG scaffolds. Biomaterials 26, 433, 2005.

30. Menard, C., Mitchell, S., and Spector, M. Contractile behavior of smooth muscle actin-containing osteoblasts in collagen-GAG matrices in vitro: implant-related cell contraction. Biomaterials 21, 1867, 2000.

31. Murphy, C.M., Haugh, M.G., and O'Brien, F.J. The effect of mean pore size on cell attachment, proliferation and migration in collagen-glycosaminoglycan scaffolds for bone tissue engineering. Biomaterials 31, 461, 2010.
32. Haugh, M.G., Murphy, C.M., and O'Brien, F.J. Novel freezedrying methods to produce a range of collagen-glycosaminoglycan scaffolds with tailored mean pore sizes. Tissue Eng Part C Methods 16, 887, 2010.

33. Kim, Y.J., Sah, R.L., Doong, J.Y., and Grodzinsky, A.J. Fluorometric assay of DNA in cartilage explants using Hoechst 33258. Anal Biochem 174, 168, 1988.

34. Jorge-Herrero, E., Fernandez, P., Turnay, J., Olmo, N., Calero, P., Garcia, R., et al. Influence of different chemical crosslinking treatments on the properties of bovine pericardium and collagen. Biomaterials 20, 539, 1999.

35. Torres, D.S., Freyman, T.M., Yannas, I.V., and Spector, M. Tendon cell contraction of collagen-GAG matrices in vitro: effect of cross-linking. Biomaterials 21, 1607, 2000.

36. Charulatha, V., and Rajaram, A. Influence of different crosslinking treatments on the physical properties of collagen membranes. Biomaterials 24, 759, 2003.

37. Pieper, J.S., Oosterhof, A., Dijkstra, P.J., Veerkamp, J.H., and van Kuppevelt, T.H. Preparation and characterization of porous crosslinked collagenous matrices containing bioavailable chondroitin sulphate. Biomaterials 20, 847, 1999.

38. Gilles, M.A., Hudson, A.Q., and Borders, C.L., Jr. Stability of water-soluble carbodiimides in aqueous solution. Anal Biochem 184, 244, 1990.

39. Ingber, D. Integrins as mechanochemical transducers. Current Opin Cell Biol 3, 841, 1991.

40. Ingber, D.E. Tensegrity: the architectural basis of cellular mechanotransduction. Annu Rev Physiol 59, 575, 1997.

41. Kim, K.M., Herrera, G.A., and Battarbee, H.D. Role of glutaraldehyde in calcification of porcine aortic valve fibroblasts. Am J Pathol 154, 843, 1999.

Address correspondence to: Fergal J. O'Brien, B.A., B.A.I., Ph.D. Department of Anatomy Royal College of Surgeons in Ireland 123 St. Stephen's Green Dublin 2 Ireland

E-mail: fjobrien@rcsi.ie

Received: October 11, 2010 Accepted: December 13, 2010 Online Publication Date: January 12, 2011 